\title{
I ndicadores de evaluación de la educación a distancia en un sistema universitario
}

\author{
(Evaluation indicators in distance education at the university system)
}

\author{
JAVIER CALLEJO, TERESA AGUADO, BELÉN BALLESTEROS, \\ INÉS GIL JAURENA Y BEATRIZ LÓPEZ \\ Universidad Nacional de Educación a Distancia (UNED) \\ Instituto Universitario de Enseñanza a Distancia (IUED)
}

\begin{abstract}
RESUMEN: La cuestión que nos planteamos es si es posible establecer un sistema único de indicadores de calidad para todas las universidades. Más allá de la particularidad de la educación a distancia, queda evidenciada la falta de un consenso sobre cuáles han de ser los objetivos de la Universidad. Los indicadores habitualmente utilizados para estimar la calidad de la enseñanza universitaria no pueden sin más ser aplicados a la modalidad universitaria a distancia. Se analiza cómo los indicadores no son unívocos ni análogos, que exigen interpretaciones y juicios diferentes cuando son aplicados a una u otra modalidad de enseñanza. Se proponen indicadores especializados que puedan tener en cuenta los aspectos específicos de la enseñanza impartida a distancia.
\end{abstract}

\section{Educación universitaria- Enseñanza a distancia- Indicadores de calidad}

\begin{abstract}
The key- question the article propose is if it is possible to stablish a unique quality indicators system for all the universities. We realize there is no a consensus about which are the objectives at the University level. Therefore, it seems lightly difficult to accept a common indicators system. The authors analyze why the indicators used to evaluate University teaching quality are not univoques or analogues, they have different meanings when applies at distance teaching. Finally, the authors propose and define some specific indicators to evaluate distance education at the University system.
\end{abstract}

University Education- Distance Teaching- Quality indicators 


\section{EVALUACIÓN DE LA UNIVERSIDAD: ¿PARA QUÉ?, ¿CÓMO?}

Desde hace algunos años, el conjunto de la Universidad pública española ha ido entrando paulatinamente en la práctica de la evaluación. En principio, la finalidad principal ha sido la propia instauración de la cultura de la evaluación en un ámbito institucional más inclinado a mostrar y mostrarse, que a ser observado y enjuiciado. De hecho, ha de reconocerse que las resistencias no han sido pocas. Se insiste en que la evaluación no es un fin en sí misma sino un medio para planificar y tomar decisiones de mejora (Escudero, 2000). Desde esta perspectiva la evaluación lleva implícita la idea de gestión de la calidad, sin tratarse tanto de una mera actividad centrada en la valoración de la efectividad y eficiencia de las instituciones, como en la constitución de una base a partir de la cual se diseñarán nuevas líneas estratégicas que supongan una propuesta de mejor.

Sin embargo, el término calidad tiene un carácter relativo en cuanto que alude a una actividad de valoración y, como tal, a una comparación en la que podrán adoptarse distintas referencias o patrones, (Municio, 2000). La adopción de criterios diferentes produce una «confusión» de términos, resultando el concepto mismo de calidad manifiestamente desdibujado. (Bricall, 1999):

- En relación con los términos de excelencia y distinción. Se considera de calidad una universidad que se distingue en algunas de sus características:los profesores, los estudiantes, los recursos financieros, sus instalaciones,su historia, su reconocimiento público.

- Como conformidad con unos estándares. La acreditación de una universidad para otorgar títulos caería dentro de este concepto.

- Adecuación a un objetivo o finalidad. Se trataría de establecer el grado de consecución de la misión y objetivos de una universidad, su eficacia enconseguir los objetivos institucionales.

- Eficiencia y el coste de la Universidad. Se implica de forma decisiva a los ámbitos de gestión y administración.

- Aptitud para satisfacer las necesidades de los usuarios, destinatarios o clientes. Esta visión de calidad, plenamente aceptada en la industria y servicios, provoca incomprensión y resistencia en la Universidad. Formularequerimientos no siempre compatibles con la idea misma de Universidad.

- Capacidad de transformación y cambio de la Universidad. Este concepto pone el énfasis en la necesidad de gestión del cambio ante las nuevas demandas de la sociedad, de mejora de procesos, de introducción de tecnologíaactual, de adaptación de los recursos humanos.

Teniendo en cuenta el carácter multidimensional de la calidad, podemos señalar la existencia de un consenso generalizado en la concreción de dos perspectivas genéricas en torno a este concepto, (Consejo de Universidades, 2000):

- Calidad intrínseca, que implica el acatamiento de las exigencias epistemológicas de una disciplina.

- Calidad extrínseca, referida al equilibrio entre la misión o misiones de la Universidad con las necesidades del entorno.

Desde este marco conceptual de referencia, la Universidad española ha optado, promovido por razones de índole formativas, políticas y socioeconómicas, por la 
implantación de un modelo de evaluación institucional que pretende aunar, aun a riesgo de imperfecciones, ambos enfoques.

Se espera que los sistemas de calidad universitaria permitan, entre otras metas (Consejo de Universidades, 1996, 2000b; Bricall, 1999):

- Promover la evaluación institucional de la calidad de las universidades.

- Elaborar metodologías homogéneas para la evaluación de la calidad integradas en la práctica vigente en la Unión Europea.

- Ofrecer información objetiva que pueda servir de base para la adopción de decisiones de las distintas organizaciones y administraciones universitarias.

Entre otras posibles acciones estarían:

- Preparar los mecanismos para la acreditación de instituciones o programas.

- Mejorar la docencia, la investigación y la administración.

- Servir de instrumento para rendir cuentas al gobierno y a la sociedad.

- Suministrar información pública y transparencia a los estudiantes y al mercado laboral.

- Operar como mecanismo para decidir una financiación diferencial de las universidades.

En el Plan Nacional de Evaluación de la Calidad de las Universidades (1996,2000) quedaban primados dos aspectos: la autoevaluación, pues importaba la implicación en el proceso evaluativo del máximo de agentes de los centros o entidades, y la publicidad de los resultados, tanto dentro de los centros, siguiendo la misma lógica de la implicación general, como al conjunto de la comunidad universitaria y, en extensión, a la sociedad, adquiriendo no pocas veces las evaluaciones realizadas un carácter modélico, ya que en muchos casos eran las primeras:la primera evaluación en la Universidad española de un tipo específico de estudios o un servicio (biblioteca, deportes, etc.).

No obstante, ahora aparece la posibilidad de una nueva etapa, admitiendo, al menos parcialmente, como superada la anterior. De hecho, el III Foro de Almagro $(7$ y 8 de mayo de 2001)1 introduce dos sesiones específicamente dedicadas a los sistemas de acreditación y a la conformación de un catálogo (no se utiliza

el término sistema de indicadores, que parece más habitual en la literatura metodológica) de indicadores para la calidad. Dos elementos que transforman radicalmente el escenario de la evaluación universitaria española y que, además, han de motivar una reflexión profunda: tanto sobre el conjunto de la política universitaria, pues parece difícil evaluar, aun cuando sea un concepto tan vaporoso como la calidad, sin un conocimiento y asunción de los fines que se proponen para el conjunto del sistema universitario público; como sobre el grado de encuentro en un conjunto de indicadores entre un tipo de universidades, las presenciales, y las que, como la UNED, son universidades con una metodología específica apoyada en la educación a distancia. Este segundo aspecto es el que centra el presente trabajo. 


\section{HACIA UN SISTEMA ÚNICO DE INDICADORES DE CALIDAD DE LA UNIVERSIDAD.}

La cuestión que nos planteamos es si es posible establecer un sistema único de indicadores de calidad para todas las universidades. Antes de introducirnos en los posibles puntos de encuentro y desencuentro específicos para un posible único sistema de indicadores de calidad de la Universidad, que recoja la enseñanza presencial como la enseñanza a distancia, conviene una mínima introducción sobre los distintos tipos de evaluación, no excluyentes entre sí, que pueden llevarse a cabo y que, por lo tanto, enfocaría el sistema de indicadores. Para algunos, tal introducción tal vez sea un paso atrás, una regresión a las discusiones iniciales -e iniciáticas- del proceso de evaluación de la Universidad española; pero parece difícil pensar en clave de indicadores si no se tiene claro el para qué de la evaluación. Es más, en toda propuesta de sistema de indicadores, ha de plantearse tanto su finalidad, como sus agentes. Es decir, no se trata sólo de un paso metodológico, sino que seguramente hay que volver a la discusión global. Junto a qué indicadores, qué procedimientos, quiénes los van a generar, quiénes a certificar, etc. Desde tal marco y siguiendo parcialmente a José-Ginés Mora (1999), cabe distinguir, al menos, tres tipos de sistemas de indicadores, que a continuación se analizan.

\footnotetext{
${ }^{1}$ Los denominados Foros de Almagro, de los que se llevan tres hasta la fecha, constituyen un periódico encuentro entre autoridades y expertos universitarios españoles alrededor de la evaluación de la calidad en la Universidad.
}

\subsection{INDICADORES DE RENDIMIENTO.}

Los que el autor (Mora, 1999), denomina indicadores de rendimiento y que directamente relaciona con los objetivos. Parece obvio que toda acción deba ser evaluada, en principio, en función de las metas que se propuso y, por lo tanto, el grado en que éstas fueron alcanzadas. De hecho, esto es lo que habría que medir en toda institución y servicio público. El problema para tal medición no es, como apunta Mora (1999), que no haya definición de objetivos. Basta un repaso a los estatutos de las distintas universidades para confirmar su existencia. Los problemas derivan de:

1. La tendencia a tratarse de unos objetivos de carácter macrosocial, frecuentemente más ligados a una retórica grandilocuente que a una vinculación concreta con acciones específicas. Por otra parte, la adecuación de las metas y planes de acción establecidos a las necesidades sociales en distintos momentos a través de la historia, supone una actitud reflexiva diacrónica que no es mantenida por la Universidad en términos generales, (Zúñiga, 1997). Pensemos por ejemplo en universidades que definen su misión como una forma de potenciación de su entorno socioeconómico y, con posterioridad, la progresiva implantación de nuevas titulaciones poco o nada guardan relación con las posibilidades laborales, sino que más bien suponen una vía de reclutamiento de la Universidad en sí. 
Con todo, la operativización de objetivos en indicadores no es ni mucho menos fácil, aun cuando tampoco imposible.

2. Difieren entre unas universidades y otras. Sólo a modo de ejemplo, cabe citar la diferencia entre el acento que algunas entidades ponen en la cuestión de la lengua autóctona (la mayor parte de las universidades inscritas en Comunidades Autónomas con lengua propia), frente a otras que se establecen como dispositivos de extensión del área cultural hispana (Universidad de Salamanca); desde las que ponen de relieve objetivos de carácter medioambiental (Universidades de Alicante, Valencia, Jaén, etc.) a las que presentan unos objetivos más centrados en la empresa y el empleo, como ocurre con las universidades politécnicas. En común, apenas queda la intención de fomentar el espíritu crítico y, claro está, la prestación de un servicio público de la calidad superior. Diferencias en los objetivos que, más allá de la reconocida idiosincrasia de cada entidad, parecen señalar las dificultades para la definición del papel de laUniversidad en las sociedades actuales. De hecho, simultáneamente al aumento de la demanda de estudios universitarios se vive una creciente deslegitimación de la Universidad, sin que tan paradójica circunstancia -al menos desde un primer acercamiento- parezca ser patrimonio exclusivo de ninguno de los países occidentales.

3. La diferencia de objetivos queda remarcada cuando entran en juego las universidades a distancia, cuyo papel tradicional en los sistemas educativos europeos occidentales ha sido -y es- facilitar el acceso a la enseñanza universitaria a sectores de población que, por diversas razones, han tenido dificultades para el mismo. Hay que tener en cuenta que, tras esta diferencia, se encuentra un debate que parece volver con fuerza: ¿son los estudios universitarios un derecho que hay que restringir a ciertas élites, ya sea por capacidad económica, laboral o, incluso, intelectual, o han de extenderse a todos aquellos que cumplan un mínimo de requisitos formativos previos? Ni que decir tiene que la apuesta por la igualdad social en los objetivos estatutarios no es prerrogativa exclusiva de las universidades a distancia. También puede encontrarse en otras universidades españolas (siempre sólo a modo de ejemplo: Universidades de Lleida o Zaragoza). Pero, como se ha señalado, es a las universidades a distancia a las que tradicionalmente el sistema educativo ha encargado tal función. Como muestra, en los Estatutos de la UNED, título preliminar (naturaleza, fines y autonomía), la primera delas funciones de la UNED que se nombra es:

«Facilitar preferentemente el acceso a la enseñanza universitaria y la continuidad de sus estudios a todas las personas que, estando capacitadas para seguir estudios superiores, no puedan frecuentar las aulas universitarias por razones laborales, económicas, de residencia o cualquiera otra de similar consideración» (Estatutos de la UNED, art. $3^{\circ}$ )

Bajando nuevamente al nivel de los indicadores, no pueden ser los mismos o tener el mismo peso, en el caso de un sistema de indicadores, según la institución tenga por misión abrir la Universidad a la sociedad, donde lo importante será calibrar el grado de tal apertura, que aquéllas que tengan por finalidad ubicarse en la punta del desarrollo científico y técnico. Seguramente todas comprendan, en mayor o menor grado, ambos tipos de objetivos; pero la cuestión está en la jerarquía que se da a los mismos. La 
pregunta es: ¿puede un sistema de indicadores común recoger tales diferencias jerárquicas en los objetivos?

Si la información sobre los indicadores con relación a objetivos la proporcionan las propias universidades, como ocurre, en mayor o menor medida, con todos los sistemas de indicadores posibles, parece que los agentes de la evaluación han de ser externos, asumiendo el papel de representantes de la sociedad a la que se rinden cuentas. El esquema es sencillo: la sociedad, a través de sus instituciones y representantes, establece unas funciones para su universidad, de manera que es la misma sociedad la que habrá de dar cuenta de su grado de satisfacción con el cumplimiento de tales funciones.

\subsection{INDICADORES DE PROCESOS}

Estarían dentro de lo que Mora señala como evaluación institucional, que es, por otro lado, la que ha centrado los esfuerzos de evaluación de la Universidad española. Los indicadores medirían aspectos que, de alguna $\mathrm{u}$ otra forma, se han ido midiendo o, al menos, recogiendo en los últimos años, pues, básicamente mide lo que puede medirse: costes, personal, resultados académicos, resultados de investigación. Otra cosa es que se esté de acuerdo en que, por ejemplo, los resultados académicos se establezcan a partir del número de titulados o, aún con mayor detalle, del número de titulados en un determinado tiempo, o las calificaciones, pues, especialmente en este último concepto, cabrían varias interpretaciones: así, por ejemplo ¿tiene más valor que la media de calificación de los titulados sea de un siete, cuando se exige una calificación de entrada de un ocho, o que tal media sea de un seis, cuando sólo se exige una calificación de entrada de un cinco? En principio, parece que el trabajo de la institución -el output en función del input- ha sido mayor en el segundo de los casos; sin embargo, se tiende a comparar desde la primera perspectiva. Cuestiones parecidas podrían discutirse con respecto a los resultados de la investigación, habitualmente concentrados en indicadores que recogen las publicaciones.

La participación de la propia Universidad como agente evaluador es mayor en este caso, adquiriendo, aun cuando sea parcialmente, el carácter de autoevaluación. La finalidad es hacer mejor las cosas de lo que se están haciendo, sin cuestionar los objetivos. Tampoco se evalúa si se logran o no.

A pesar de las evidentes limitaciones de este tipo de evaluación, especialmente a la hora de pensar en la creación de un sistema de indicadores comunes, hay que destacar su relevancia en el funcionamiento cotidiano de las instituciones y la trascendencia que pueden adquirir algunos indicadores del output, como son: empleabilidad de los titulados, impacto científico de la investigación o impacto socioeconómico de la actividad desarrollada por la universidad, tanto científica como educativa.

\subsection{INDICADORES DE PRODUCTOS}

Reconociéndose a la lógica de la competencia -y, en definitiva, el mercado como principal impulsora de las acciones de la Universidad, se trataría de buscar indicadores que reflejasen la evaluación de los distintos productos universitarios, especialmente titulaciones y cursos, por tal mercado y, sobre todo, de sus sujetos soberanos: los clientes y usuarios, aun cuando tales conceptos, aplicados al ámbito universitario, requieran una definición previa. Ocuparían un lugar central las muestras del grado de 
satisfacción por parte de graduados y empleadores con respecto a los servicios y productos específicos ofertados, así como de los rasgos y características de éstos; pero también estaría incluida la opinión y percepción del conjunto de la sociedad.

En la medida que se utilicen instrumentos de medición de la satisfacción comunes, la comparación entre unas instituciones y otras quedaría facilitada. De hecho, para otros ámbitos del mercado y el consumo, tales instrumentos tienen una relativamente larga experiencia. Piénsese, por ejemplo, en los estudios ad hoc sobre sistemas de imágenes de marca o sistemas de imágenes de productos. Por otro lado, cabría también la posibilidad de un estudio anual para el conjunto de la Universidad española, con una importante base muestral, haciendo la comparación aún más directa. Tal vez un modelo a seguir, expuesto aquí sólo a modo de ejemplo ilustrativo en otro campo, es el del Estudio General de Medios (AIMC), realizado para un buen número de medios de comunicación españoles. Aun cuando las diferencias son amplias, pues aquí se centraría no tanto en el uso-compra de la oferta, como en el grado de satisfacción de tal oferta, podría tomarse su carácter general y relativamente externo a las propias entidades evaluadas, aun cuando se encuentren representadas en sus distintos comités, noquedando exentos los continuos debates y discusiones, tanto metodológicas como conceptuales.

Los tres tipos de posibles sistemas de indicadores tienen distintas pautas. En definitiva, evalúan cosas distintas puesto que implican modelos de Universidad distintos. Cada uno con sus evidentes ventajas; pero también con sus peligros. El primer modelo puede caer en una excesiva dependencia y subordinación del sistema político y, lo que es peor, de los gobernantes particulares en cada momento. El peligro del segundo puede encontrarse en la cultura de las organizaciones universitarias. De hecho y a pesar de que es el resultado de una clara convicción de la necesidad de ir mejorando paulatinamente el servicio público dado por estasinstituciones, tiene todavía algo de burocracia vertical, con cuidadosa separación entre las distintas categorías, condensación de lo racional en razón burocrática y, en general, poco flexible2. También algo de vacuna, de autocontrol para que el control no sea externo. De hecho, este argumento ha sido repetidamente utilizado en los distintos Foros de Almagro, como arma disuasoria frente a quienes todavía se encontrasen dubitativos ante los beneficios de la evaluación: establecer mecanismos de autocontrol, para evitar que se impongan «desde fuera». Por último, sobre el tercer modelo ha de indicarse que no siempre el mercado se rige por criterios de excelencia, pudiendo concentrarse las mejores imágenes de «producto» y «marca» no en quienes lo hagan mejor sino en aquellas instituciones que, por vía publicitaria $u$ otra cualquiera, mejor sepan ofrecer su imagen al público en general. Además, cuando un instrumento metodológico de las características del propuesto, se constituye en un dispositivo que certifica el mercado, se convierte en constante receptor de críticas a posteriori entre quienes no salen bien parados en los periódicos resultados.

\footnotetext{
${ }^{2}$ Para el concepto burocracia vertical, frente a empresa horizontal y flexible, véase Manuel Castells, La era de la información (Vol.1. La sociedad red). Madrid: Alianza, 2000, pág. 215 (2 $2^{\mathrm{a}}$. ed.).
}

\section{INDICADORES DE CALIDAD EN LA ENSEÑANZA UNIVERSITARIA A DISTANCIA.}


La educación universitaria a distancia también se encuentra en posiciones distintas con respecto a unos y otros. En principio, parece más difícil la integración de su particularidad en un sistema común en los tipos a), pues sus objetivos principales y básicos tienden a ser distintos a los de las otras universidades, y b), ya que los procesos utilizados, especialmente los relativos a la metodología de la enseñanza a distancia, son también distintos.

Más allá de la particularidad de la educación a distancia, queda evidenciada la falta de un consenso sobre cuáles han de ser los objetivos de la Universidad. Incluso dentro de los muros de la Universidad, es considerable la ambigüedad sobre el papel de las universidades. Como no los hay de manera evidente, se hace hincapié en los procesos, en el cómo, y en indicadores del cómo, subyaciendo la idea, más implícita que explícita en las manifestaciones públicas, de que la Universidad está gastando cantidades irracionales de dinero, luego hay que: a) reducir el gasto, pudiéndose llegar a una especie de ranking de supervivencia, de manera que ocuparán las primeras plazas quienes sobrevivan con menos dinero por alumno; b) racionalizar el gasto; pero, como no hay objetivos claros, se hace hincapié en los medios.

En definitiva, antes de iniciar la discusión sobre sistemas o catálogos de indicadores, queda responder a la pregunta sobre qué es lo que hay que evaluar,lo que arrastra otras preguntas: ¿es función de la Universidad generar el mayor número de licenciados con la gente que acude a ella? ¿es función de la Universidad crear el mayor número de titulados en el menor tiempo posible? ¿es su función certificar la capacidad profesional de los licenciados y, a su vez, establecer los criterios de tal certificación? ¿es su función generar mano de obra para las demandas de los empresarios, sin entrar en las características de tal demanda? ¿es su función generar conciencia crítica y percepción global de las cosas? ¿generar nuevos saberes y conocimiento, lo que parece más ligado a la investigación que a la formación de profesionales?. Como puede verse las funciones pueden ser muy distintas y, cuando se trata de racionalizar recursos, hasta pueden ser opuestas. Seguramente porque bajo la denominación de Universidad se encuentran múltiples actividades y finalidades, incluso dentro de una misma institución.

Como se ha visto, toda universidad tiende a presentarse con características particulares. Pero el caso de la específica metodología de la enseñanza a distancia refuerza el grado de particularidad. Es más, se distancia de indicadores pueden ser habituales o «normales» en la evaluación de la enseñanza universitaria presencial; mientras que pone el acento en otros. Así: «muchos de los instrumentos de medida utilizados para medir la educación tradicional no se transfieren bien a las prácticas de la educación a distancia» (Hidalgo, 1999). Los indicadores habitualmente utilizados para estimar la calidad de la enseñanza universitaria no pueden sin más ser aplicados a la modalidad universitaria a distancia. Es preciso considerar que no son unívocos ni análogos, que exigen interpretaciones y juicios diferentes cuando son aplicados a una u otra modalidad de enseñanza. Además, es necesario definir indicadores especializados que puedan tener en cuenta los aspectos específicos de la enseñanza impartida.

\subsection{INDICADORES CONVENCIONALES APLICADOS A LA ENSEÑANZA A DISTANCIA.}

Adaptar los indicadores convencionales a la modalidad a distancia supone tener en cuenta algunos efectos posibles. A veces, son indicadores que simplemente no pueden 
ser, sin más, aplicados a la enseñanza a distancia. Algunos de ellos pueden ser transferidos, pero exigen juicios e interpretaciones específicos. En otros casos, se requieren indicadores específicos para la modalidad de enseñanza a distancia.

Entre los indicadores habitualmente aceptados en la enseñanza tradicional de difícil aplicación en la enseñanza universitaria a distancia se encuentran:

- Los relativos a la demanda, pues tiende a satisfacerse casi toda la demanda, especialmente si se trata de universidades abiertas. Por otro lado, de una necesidad, la falta de plazas que ofrecer a una demanda, se tiende a hacer virtud bajo el concepto de selección y remarcando la calidad de los alumnos seleccionados a partir de las notas mínimas con las que acceden al centro universitario.

- En este sentido, indicadores que valoran la proporción entre los alumnos inscritos en primera opción sobre la oferta de plazas o la proporción entre alumnos de nuevo ingreso en primera opción sobre el total de nuevo ingreso, carecen de interés para el caso de la UNED y la mayor parte de las universidades abiertas, donde no existe esta priorización de opciones parala elección de estudios, como tampoco número clausus.

- La consideración de la nota de selectividad como indicador nos lleva a plantearnos ¿qué calidad de la Universidad se indica con las calificaciones que los alumnos obtienen fuera de la misma? Es más, el trabajo del centro universitario habría que evaluarlo no tanto a partir de la calificación de entrada, sino de la comparación de la calificación de salida con la de entrada, siempre en el supuesto de que pudiera establecerse una conexión entre el rendimiento logrado en niveles educativos precedentes y el alcanzado en la Universidad. Si por el contrario se llegara a fundamentar la incompatibilidad entre estas dos realidades, en función de criterios de especialización, tipo de evaluación, compatibilidad con otras actividades, entre otros, ni siquiera relativizar la calificación de salida con respecto a la de entrada tendría sentido.

- La movilidad de los alumnos, puesto que las universidades a distancia son, en la mayoría de los casos, dispositivos de los sistemas educativos para paliar, en la medida de lo posible, las dificultades y costes para tal movilidad. Por otro lado, con el impulso de este concepto, se está prefigurando un tipo de alumno: joven, sin obligaciones laborales ni familiares, bastante alejado del perfil característico del alumno de la UNED y de la mayor parte de las instituciones universitarias a distancia del mundo desarrollado.

En cualquier caso el objetivo del análisis de la movilidad del alumnado queda un tanto encubierto. ¿Se pretende que cada Universidad oferte la mayor gama de titulaciones o que ofrezca aquellas titulaciones que más se adecuan a su entorno sociolaboral?, ¿cómo debe ser interpretada, por tanto, la movilidad?

- La aplicación de indicadores de tipo organizativo, tales como la proporción de grupos grandes o pequeños en la titulación, o proporción estudiante/ profesor, teniendo en cuenta la estructura organizativa de nuestra Universidad, -Sede Central y Centros Asociados- provoca una ambigüedad en la interpretación. 
- Los indicadores relativos a la interactividad presencial en el aula, como evaluación de calidad de exposición, actividades en clase, etc

- Los relativos a una especie de rendimiento inmediato, acumulación de créditos académicos acumulados en el momento de la matrícula o eficacia productiva, es decir, aquellos que valoran la obtención del título en el menor tiempo posible. En primer lugar, hay dificultades metodológicas para admitir como dedicación lectiva del alumnado indicadores como, por ejemplo, el número de asignaturas o parte de asignaturas en las que se matricula. Por otro, hay que tener en cuenta que el alumnado principal de la enseñanza a distancia comparte los estudios con obligaciones laborales (70,3\%) (Callejo, Calcerrada, y García Cuesta, 2001) o incluso, como viene dándose en los últimos años, con otros estudios, circunstancias que le impiden abordar las mismas trayectorias que al alumno tipo considerado en el catálogo de indicadores que se está elaborando desde el Consejo de Universidades (Consejo de Universidades, 2001). A lo sumo, en caso de comparación estandarizada, lo que comúnmente ya se conoce como ranking, debieran compararse universos semejantes, como, por ejemplo, alumnos entre el mismo intervalo de edad (18-24 años, que en la UNED suponen un $21 \%$ del alumnado), de no ser posible la comparación entre grupos de alumnos que parten de unas mismas situaciones iniciales. En concreto en la UNED hay dos intervalos de edad donde se concentra el alumnado. El primero está formado por jóvenes entre 20 y 29 años (46,2\%), y el segundo por adultos jóvenes entre 30 y 39 años (38,5\%) (Callejo, Calcerrada y García Cuesta, 2001).

De no considerarse de este modo, los indicadores referidos a la tasa de rendimiento, éxito, tasa de graduación, dedicación lectiva del alumnado, así como la duración media de los estudios no resultan significativos como criterios de comparación entre las universidades.

- Indicadores que hacen referencia a la capacidad de la Universidad en la preparación de sus alumnos para la inserción laboral. Siendo coherentes con lo expuesto en puntos anteriores, si el alumno de la UNED se encuentra ejerciendo ya un puesto de trabajo, no resulta válido pensar que entre sus motivos para iniciar unos estudios universitarios esté la necesidad de formación para encontrar un empleo o entrar en el mercado laboral. Si bien es cierto que en algunos casos el estudio de una carrera en la UNED puede servir como vía de promoción profesional, tampoco hay que ignorar el hecho de que una buena parte de nuestro alumnado estudian por razonesdistintas a las profesionales.

Por todo ello, indicadores como la tasa de ocupación de los graduados universitarios, población ocupada con estudios universitarios, satisfacción con el empleo y, a más, satisfacción con los estudios (siendo considerado desde una perspectiva restrictiva en la que se presta especial atención a las posibilidades que brinda para la consecución de un empleo, ignorando otros aspectos que conciernen a un término tan global como es el de «satisfacción»), pierden significatividad al ser aplicados en nuestro sistema universitario. Se podría pensar en una solución en la que se analizaran estos indicadores tomando como base de referencia el número de alumnos que han intentado la inserción/promoción en sus puestos de trabajo, en lugar de establecer esta proporción sobre el total del alumnado egresado. 
Indicadores de recursos referidos al gasto corriente por alumno matriculado, donde en el caso de la UNED se deja sin contemplar aquellos costes de la edición y distribución de material didáctico así como otros recursos complementarios en diversos soportes. La omisión en este aspecto supone una negación de la especificidad de nuestra metodología a distancia,además de las repercusiones que conlleva a efectos de dotación presupuestaria.

\subsection{INDICADORES ESPECÍFICOS DE LA ENSEÑANZA A DISTANCIA.}

Por otro lado, se encuentran una serie de identificadores o características que intentan recoger el quehacer de la enseñanza a distancia que no siempre tienen cabida en los sistemas de indicadores más tradicionales. Sobre todo los destinados a recoger la evaluación de:

- El perfil de nuestro alumnado en cuanto a aspectos académicos y profesionales se refiere, identificando por otra parte aquellos grupos específicos (alumnos en el extranjero, alumnos en prisiones, etc.) que subrayan en mayor medida el carácter social de nuestra institución.

- Materiales didácticos utilizados, especialmente escritos; pero también en otros soportes: Internet, vídeo, cassettes, etc.. Al respecto, parece que esta cuestión no ha de tener problemas de integración en sistemas de indicadores más generales, pues, por ejemplo, pueden evaluarse los textos o manuales utilizados en una asignatura. Sin embargo, hay que tener en cuenta que con los soportes digitales la evaluación de los materiales va más allá de lo expuesto, abriéndose también a su utilización en otros procesos de la enseñanza: participación de los alumnos, grado de interactividad, vínculos con otros aspectos paralelos de la materia o disciplina abordada, gestión de investigaciones, etc.

- Vías y procedimientos de distribución, medios de comunicación: radio, televisión, videoconferencia, Internet, etc.

- Tutorías presenciales, más allá de recoger su existencia. De hecho, los aspectos de la tutoría en la enseñanza a distancia susceptibles de evaluación son múltiples: desde la adecuación del tutor a la programación seguida, pasando, entre otros, por los horarios o la capacidad de respuesta a las consultas.

- Tutorías a distancia (respuestas de los profesores encargados de las materias o asignaturas), ya sea a través de medios más tradicionales, postal, como más modernos: teléfono, telemáticos, etc.

- La formación de nuestros profesores tutores.

- Los recursos físicos y materiales ofrecidos por el Centro Asociado.

- La vinculación de los alumnos con la institución. En la enseñanza tradicional se hace hincapié en que los alumnos abandonen lo antes posible las aulas, considerándose como fracaso o abandono lo que se aleje de tal horizonte. Sin embargo, en la enseñanza a distancia, como en las ofertas de consumo 
habituales, la vinculación con los alumnos o los «clientes» o «usuarios», siguiendo la terminología que tiende a imponerse, es un aspecto importante que parece indicar algún grado de calidad: en la medida que alguien sigue vinculado a una oferta, algún grado de satisfacción encuentra. Por ello, conceptos como tasa de retención del alumnado, refiriéndosea la capacidad de mantener su esfuerzo, cobran relevancia.

Por otra parte, al margen de esta dicotomía que venimos estableciendo entre el sistema presencial y el sistema a distancia, la propuesta de algunos indicadores no deja de sembrar ciertas dudas sobre su posible interpretación y utilización: ¿hasta qué punto cabría defender en el conjunto de la Universidad española la especialización del docente tomando como referencia la temática investigada en su tesis doctoral más que la adecuación de su docencia a su proyecto docente?, o también ¿por qué el porcentaje de profesores permanentes es interpretado como un indicador de calidad más que como un signo de la política de estabilización seguida en la Universidad

En términos generales podemos afirmar que una crítica constructiva de una propuesta de indicadores precisa una información detallada sobre algunas cuestiones previas que posibiliten un diálogo efectivo sobre los mismos, sobre sus aplicaciones e interpretaciones. En este sentido, habría que exigir la explicitación de aspectos diversos: validez de los indicadores, finalidad, base teórico-empírica en la que se sustentan, formas de combinación y ponderación de los indicadores para integrar una valoración de conjunto, entre otros aspectos.

La mayor parte de los estudiosos que han abordado la evaluación de las enseñanzas universitarias a distancia han subrayado la dificultad, cuando no directamente la imposibilidad, de una evaluación conjunta con las universidades de enseñanza tradicional. Sin embargo, las propias transformaciones que experimentan las universidades presenciales, con la incorporación de otras tecnologías en el desarrollo de sus proyectos educativos, apuntan hacia cierta convergencia entre uno y otro tipo de instituciones de enseñanza superior. Ahora bien, en muchos aspectos se trata más de una aproximación tecnológica, más que metodológica, de la enseñanza tradicional a la enseñanza a distancia, dejando a un lado los aspectos relativos al papel de esta enseñanza en el conjunto del sistema educativo y, en especial, a la apuesta -política- por la apertura de los estudios universitarios a las distintas capas de la sociedad.

En un fututo próximo la aplicación de un sistema de indicadores como medida de control de calidad de la universidad puede ser un hecho. Sin embargo, la evidencia acerca de la sensibilidad y efectividad de este modelo de evaluación de la calidad está aún por demostrarse. La elaboración de un sistema de indicadores y su aplicación correspondiente puede ser útil y aportar información significativa pues obliga a la Universidad a medir de forma crítica aspectos básicos de su labor. Sin embargo, puede terminar siendo un mero ejercicio burocrático de acumulación de documentación y datos sin repercusión en la mejora de los procesos quetienen lugar como parte de la labor de enseñanza, investigación y gestión. 


\section{BIBLIOGRAFÍA}

AGUADO, T.; GIL JAURENA, I.; HERRAZ M. Y LÓPEZ, B. (2000). Evaluación de la enseñanza universitaria. La UNED en el Plan Nacional de Evaluación de las Universidades en España, en Revista Iberoamericana de Educación a Distancia (RIED) (en prensa).

ARNOLD, R. (1999). Preliminares sobre la relevancia didáctica de cercanía y distancia, en Revista Iberoamericana de Educación a Distancia (RIED), 2 (1), 61-72.

BRICALL, J.M. (2000). Cap. VII-Calidad y acreditación, en Bricall, J.M. Informe Universidad 2000 (Informe Bricall). Madrid: MEC-Consejo de Universidades, 359-391.

CALLEJO, J.; CALCERRADA, D. y GARCÍA CUESTA, S. (2001). Perfiles y opiniones de los alumnos de la UNED. Curso 1997-98. Colección Varia, Madrid: UNED.

CASTELLS, M. (2000). La era de la información (Vol.1. La sociedad red). Madrid: Alianza, (2a . ed.).

CONSEJO DE UNIVERSIDADES (1996). Guía de Evaluación del Plan Nacional de Evaluación de la Calidad de las Universidades. Primera convocatoria. Madrid:

Secretaría General del Consejo de Universidades.

CONSEJO DE UNIVERSIDADES (1999a). Informe sobre los resultados de la primera convocatoria del Plan Nacional de Evaluación de la Calidad de las Universidades. Madrid: Secretaría General del Consejo de Universidades.

CONSEJO DE UNIVERSIDADES (1999b): Guía de Evaluación del Plan Nacional de Evaluación de la Calidad de las Universidades. Tercera convocatoria. Madrid:

Secretaría General del Consejo de Universidades.

CONSEJO DE UNIVERSIDADES (2000a). Plan Nacional de Evaluación de la Calidad de las Universidades. Informe Anual de la Segunda Convocatoria. Madrid: Secretaría General del Consejo de Universidades.

CONSEJO DE UNIVERSIDADES (2000b). Guía de Evaluación del Plan Nacional de Evaluación de la Calidad de las Universidades. Cuarta convocatoria. Madrid: Secretaría General del Consejo de Universidades. 
CONSEJO DE UNIVERSIDADES (2001). Borrador del catálogo de indicadores del sistema universitario público español. Disponible en:

http://www.mec.es/consejou/indicadores/index.html [2 de Marzo de 2001]

ESCUDERO ESCORZA, T. (2000). La evaluación y mejora de la enseñanza en la Universidad: otra perspectiva. Revista de Investigación Educativa, 18 (2), 405-416.

HIDALGO MOLINA, G. (1999). Hacia una identificación de indicadores de calidad Específicos para la educación a distancia: el caso de la UNED de Costa Rica. Revista Iberoamericana de Educación a Distancia, 2 (1), 73-94.

MORA, José-Ginés (1999). Indicadores y decisiones en las universidades, en Consejo de Universidades. Indicadores en la Universidad: información y decisiones. Plan Nacional de Evaluación de la Calidad de las Universidades. Madrid: Secretaría General Técnica del Consejo de Universidades, 19-29.

MUNICIO, P. (2000). Evaluación de la calidad, en R. Pérez Juste, F. López Rupérez, M ${ }^{a}$ D. Peralta Ortiz y P. Municio Fernández, Hacia una educación de calidad. Gestión, instrumentos y evaluación. Madrid: Narcea.

ZÚÑIGA, R. (1997). La evaluación en la acción docente, en P. Apodaca y C. Lobato (Eds.), Calidad en la Universidad: Orientación y evaluación. Barcelona: Laertes.

\section{PERFIL DE LOS AUTORES}

Javier Callejo Gallego es Director Adjunto del Instituto Universitario de Educación a Distancia (UNED). Doctor en Sociología y licenciado en CC. de la Información (Periodismo). Profesor Titular de Técnicas de Investigación Social en la Facultad de CC. Políticas y Sociología de la UNED.

Correo electrónico: mcallejo@poli.uned.es This email address is being protected from spam bots, you need Javascript enabled to view it

Teresa Aguado Odina es Directora de la Unidad Técnica de Evaluación Institucional del Instituto Universitario de Enseñanza a Distancia (IUED) de la UNED. Profesora Titular de Universidad en el Dpto. de Métodos de Investigación y Diagnóstico en Educación, de la Facultad de Educación de la UNED.

Unidad Técnica de Evaluación Institucional IUED (UNED)

Ciudad Universitaria $\mathrm{s} / \mathrm{n}$

28040 Madrid (España)

Tfno: +34 913987784

Correo Electrónico: maguado@edu.uned.es This email address is being protected from spam bots, you need Javascript enabled to view it 
Belén Ballesteros Velázquez es Profesora Titular interina en el Dpto. de Métodos de Investigación y Diagnóstico en Educación,de la Facultad de Educación de la UNED. Colabora como asesora en la Unidad Técnica de Evaluación Institucional del IUED.

Correo electrónico: bballesteros@edu.uned.es This email address is being protected from spam bots, you need Javascript enabled to view it

Inés Gil Jaurena es becaria en la Unidad Técnica de Evaluación Institucional del IUED de la UNED. Licenciada en Psicopedagogía por la Universidad de Navarra. Especialista en Programas de Intervención Psicológica en Contextos Educativos por la Universidad Complutense de Madrid.

Correo Electrónico: inesgj@bec.uned.es This email address is being protected from spam bots, you need Javascript enabled to view it

Beatriz Soledad López Pérez es becaria en la Unidad Técnica de Evaluación Institucional del IUED de la UNED. Licenciada en Psicología por la Universidad Complutense de Madrid. Desde 1989 trabaja en temas relacionados con evaluación y metodología, colaborando en ocasiones con organismos del Ministerio de Educación y Ciencia (CIDE, INCE, GSEE, UNED) y entidades privadas.

Correo Electrónico: blopez@,bec.uned.es This email address is being protected from spam bots, you need Javascript enabled to view it 